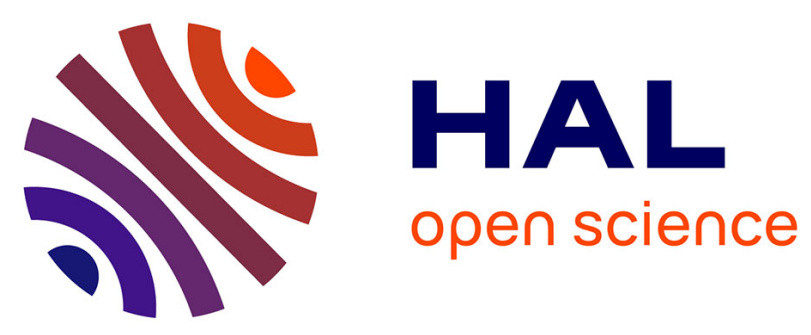

\title{
Chauffage solaire des gaz
}

F. Genneviève, G. Olalde, M. Daguenet

\section{To cite this version:}

F. Genneviève, G. Olalde, M. Daguenet. Chauffage solaire des gaz. Revue de Physique Appliquée, 1980, 15 (5), pp.985-994. 10.1051/rphysap:01980001505098500 . jpa-00244815

\section{HAL Id: jpa-00244815 https://hal.science/jpa-00244815}

Submitted on 1 Jan 1980

HAL is a multi-disciplinary open access archive for the deposit and dissemination of scientific research documents, whether they are published or not. The documents may come from teaching and research institutions in France or abroad, or from public or private research centers.
L'archive ouverte pluridisciplinaire HAL, est destinée au dépôt et à la diffusion de documents scientifiques de niveau recherche, publiés ou non, émanant des établissements d'enseignement et de recherche français ou étrangers, des laboratoires publics ou privés. 


\title{
Chauffage solaire des gaz
}

\author{
F. Genneviève, G. Olalde et M. Daguenet \\ Laboratoire de Thermodynamique et Energétique, Université de Perpignan, 66025 Perpignan Cedex, France
}

(Reçu le 3 octobre 1979, révisé le 11 février 1980, accepté le 14 février 1980)

\begin{abstract}
Résumé. - Les auteurs calculent numériquement les répartitions de température d'un gaz circulant en convection forcée à travers une cavité poreuse soumise à un rayonnement solaire concentré. Ils étudient l'influence des divers paramètres du système et comparent ses performances énergétiques à celles obtenues par le dispositif étudié par G. Olalde, M. Daguenet et J. L. Peube.
\end{abstract}

\begin{abstract}
The authors calculate numerically the profiles of temperatures of a gas circulating in forced convection through a porous cavity open to a concentrated solar radiation. They study the influence of some parameters of the system and compare their apparatus to the one studied by G. Olalde, M. Daguenet and J. L. Peube.
\end{abstract}

\section{Tableau des notations}

$A$ : surface effective pour les transferts théoriques par unité de volume (pour des sphères $(1-\xi) / \tau)$.

$C_{\mathrm{p}}$ : chaleur spécifique du gaz à pression constante.

$C_{\mathrm{s}}$ : chaleur spécifique du solide.

$D$ : diamètre des particules.

$h_{\mathrm{c}}$ : coefficient moyen de transfert de chaleur solidegaz.

$L \quad$ : longueur du matériau poreux.

$r$ : coordonnée radiale (dans le cas cylindrique) comptée positivement à partir de la face intérieure du cylindre vers le sein du matériau.

$r_{0}$ : rayon intérieur du cylindre.

$r_{\mathrm{s}}$ : rayon extérieur du cylindre.

$r^{+}$: coordonnée radiale adimensionnelle. $r^{+}=r / D$ (dans le cas cylindrique).

$R$ : coordonnée radiale (dans le cas sphérique) comptée positivement à partir de la face intérieure de la sphère vers le sein du matériau.

$R_{0}$ : rayon intérieur de la sphère.

$R_{\mathrm{s}}$ : rayon extérieur de la sphère.

$R^{+}$: coordonnée radiale adimensionnelle $R^{+}=R / D$ (dans le cas sphérique).

$T_{0}$ : température de la face du solide frappée par le rayonnement incident.

$T$ : température du solide.

$T^{+}$: température adimensionnelle du solide.

$v$ : composante moyenne de la vitesse du gaz suivant la coordonnée $x$ (cas rectangulaire), $r$ (cas cylindrique), $R$ (cas sphérique). $v_{0}$ : vitesse du gaz dans le solide supposé vide $v_{0}=\xi v$.

$x$ : coordonnée axiale (dans le cas rectangulaire) comptée positivement à partir de la face du solide frappée par le rayonnement incident vers le sein du matériau.

$x^{+}$: coordonnée axiale adimensionnelle $x^{+}=x / D$ (dans le cas rectangulaire).

$\alpha \quad$ : facteur d'absorption du solide.

$\alpha_{\mathrm{a}}$ : facteur d'absorption apparent d'une cavité.

$\varepsilon \quad$ : facteur d'émission du solide.

$\varepsilon_{\mathrm{a}}$ : facteur d'émission apparent d'une cavité.

$\xi \quad$ : porosité.

$\tau$ : longueur caractéristique de forme des grains ( $\tau=D / 6$ pour des sphères).

$\varphi$ : flux solaire incident.

$\lambda_{\mathrm{f}}$ : conductivité thermique du gaz.

$\lambda_{\mathrm{s}}^{*}$ : conductivité thermique apparente du solide.

$\sigma \quad$ : constante de Stefan.

$\mu \quad$ : viscosité dynamique du gaz.

$\rho \quad$ : masse volumique du gaz.

$\rho_{\mathrm{s}}$ : masse volumique du solide.

$\theta \quad:$ température du gaz.

$\theta_{\mathrm{a}}$ : température ambiante (température d'entrée du gaz dans le matériau).

$\theta^{+}$: température adimensionnelle du gaz.

$N u$ : nombre de Nusselt $\frac{h_{\mathrm{c}} D}{\lambda_{\mathrm{f}}}$.

$\operatorname{Pr}:$ nombre de Prandtl $\mu C_{\mathrm{p}} / \lambda_{\mathrm{f}}$.

$R e$ : nombre de Reynolds $D v_{0} \rho / \mu$. 
1. Introduction. - Le chauffage des gaz trouve, dans toutes les plages de températures, de multiples applications. Citons, par exemple, le séchage, la distillation, les réacteurs chimiques.

Les gaz sont, en général, peu conducteurs de la chaleur et absorbent peu les rayonnements de courte longueur d'onde comme ceux émis par le soleil. Il s'ensuit que le choix d'un matériau poreux comme élément de captation, de conversion, d'échange est approprié puisqu'il leur offre une grande surface de contact.

Le chauffage d'un gaz circulant en convection forcée à travers un matériau poreux soumis à un rayonnement solaire concentré a déjà été étudié par G. Olalde, M. Daguenet et J. L. Peube [1]. Cette étude a été réalisée dans le cas d'un cylindre poreux

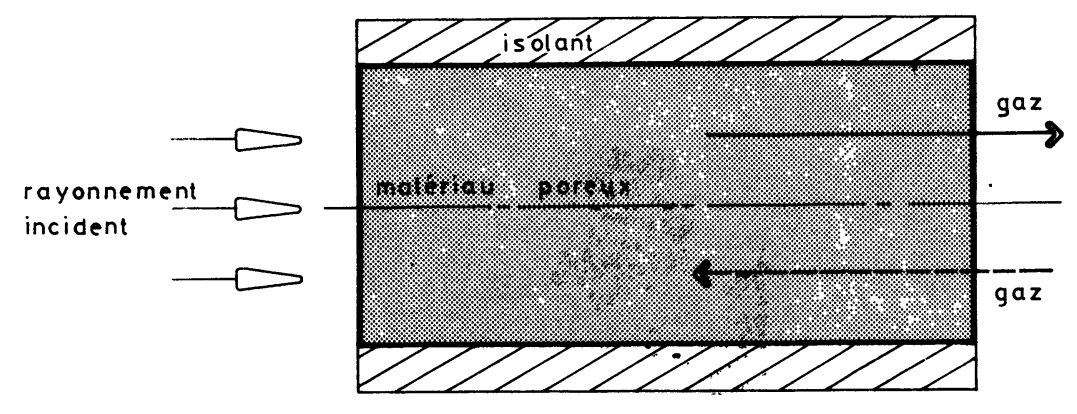

Fig. 1a. - Schéma de principe du cas rectangulaire.

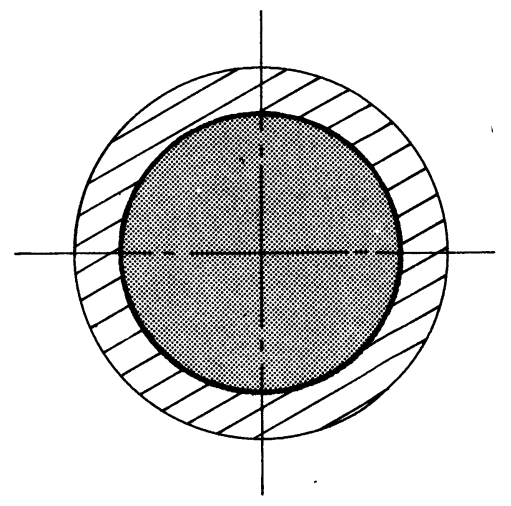

[Rectangular case model.]
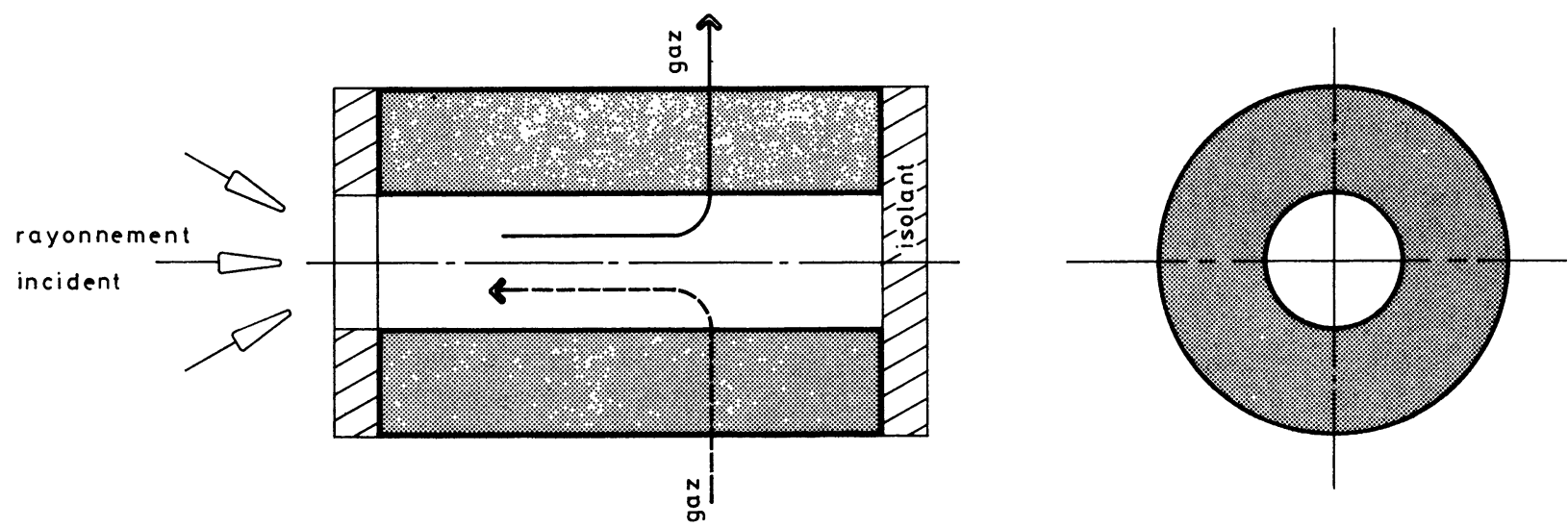

Fig. 1b. - Schéma de principe du cas cylindrique.

[Cylindrical case model.]
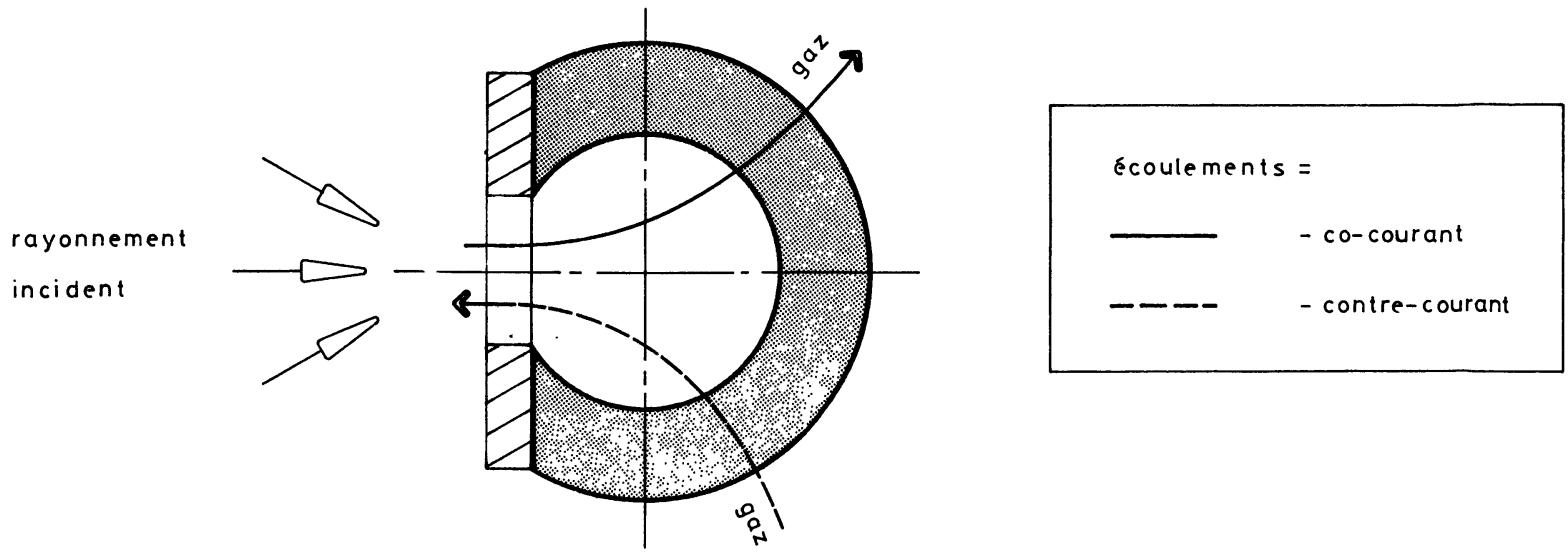

Fig. 1c. - Schéma de principe du cas sphérique.

[Spheric case model.] 
plein dont une section droite est frappée par le rayonnement solaire, le gaz circulant axialement (Fig. 1a).

Notre étude constitue un prolongement de ce travail. La géométrie du matériau poreux est différente : c'est une cavité (cavité cylindrique (Fig. 1b) ou cavité sphérique (Fig. 1c)). Le rayonnement solaire incident pénètre par l'ouverture de la cavité et subit plusieurs réflexions et absorptions sur les parois intérieures. Le gaz circule radialement.

Le système étudié par G. Olalde, M. Daguenet et J. L. Peube [1] a l'avantage d'être facile à mettre en œuvre mais il présente l'inconvénient de perdre par réémission sur la face chauffée une partie importante du rayonnement incident. C'est pourquoi nous nous intéressons aux cavités poreuses qui, elles, minimisent les pertes d'énergie par réémission.

Précisons que le milieu poreux étudié est fixe, qu'il n'existe aucun phénomène de fluidisation et que le gaz est chimiquement inerte.

Nous calculons par une méthode numérique les répartitions de températures dans le solide et dans le gaz à travers le matériau poreux en fonction de tous les paramètres du système.

Une telle étude permet de prédire les conditions expérimentales nécessaires pour porter un gaz à une température donnée, de préciser les conditions dans lesquelles le transfert de chaleur est optimal.

Le calcul de la quantité d'énergie rayonnante nécessaire pour obtenir un profil donné nous permet d'une part de définir complètement les conditions expérimentales permettant d'obtenir ce profil et d'autre part de comparer les performances énergétiques des cavités à celles obtenues par le dispositif étudié par G. Olalde, M. Daguenet et J. L. Peube [1].

2. Mise en équation du problème et hypothèses. L'évaluation du bilan de chaleur dans le milieu poreux doit tenir compte de la nature des deux phases du système : solide et gaz en examinant les contributions des trois modes fondamentaux de transfert de chaleur : conduction, convection et rayonnement [2-4].

Hypothèses de travail :

Les milieux poreux que nous considérons sont des empilements compacts de sphères identiques distribuées régulièrement.

a) Le matériau solide est homogène.

b) Le gaz est transparent au rayonnement.

c) Les propriétés physiques du gaz et du solide sont constantes.

d) Le régime est permanent.

e) L'écoulement est unidimensionnel : pour que l'hypothèse de l'écoulement unidimensionnel soit valable quelle que soit la géométrie, l'échantillon doit être isolé thermiquement à sa périphérie de maniẹre à réaliser une enceinte adiabatique. $f$ ) Le milieu poreux est contenu dans un récipient de dimension suffisante pour que les effets des parois latérales puissent être négligés.

Compte tenu des hypothèses précédentes, les équations de transfert sont obtenues en combinant les différents mécanismes des trois modes fondamentaux suivant des schémas série et parallèle. Nous écrivons :

$$
\begin{gathered}
\lambda_{\mathrm{s}}^{*}(1-\xi) \nabla^{2} T-A h_{\mathrm{c}}(T-\theta)=0 \\
\lambda_{\mathrm{f}} \xi \nabla^{2} \theta-\xi C_{\mathrm{p}} \operatorname{div}(\rho \mathbf{v} \theta)+A h_{\mathrm{c}}(T-\theta)=0 .
\end{gathered}
$$

Il est important de noter que le coefficient d'échange de chaleur $h_{\mathrm{c}}$ entre le gaz et le solide dépend ici de la vitesse du fluide, selon une relation inconnue, fonction de toutes les caractéristiques du système. Ceci nous oblige au stade du calcul numérique à utiliser une relation empirique pour déterminer les profils de températures [5].

$$
\begin{aligned}
& \text { Pour } 0<\left(\frac{1-\xi}{\xi^{3}}\right) R e<600 \\
& N u=2,62 \times 10^{-4}\left(\frac{1-\xi}{\xi^{3}}\right)^{1,6} R e^{1,6} . \\
& \text { Pour }\left(\frac{1-\xi}{\xi^{3}}\right) R e>600 \\
& N u=6,6 \times 10^{-2}\left(\frac{1-\xi}{\xi^{3}}\right)^{0,75} R e^{0,75} .
\end{aligned}
$$

Cette corrélation du nombre de Nusselt en fonction du nombre de Reynolds est utilisée quelle que soit la géométrie considérée.

Nous complétons les équations différentielles précédentes par des conditions aux limites qui dépendent du sens de circulation du gaz par rapport au sens du flux d'énergie. Nous appelons cas co-courant le cas où le gaz circule dans le sens du flux d'énergie et cas contre-courant celui où il circule en sens contraire.

Les conditions aux limites sont choisies aux sections d'entrée et de sortie du gaz dans le matériau :

- dans le cas co-courant

à l'entrée du gaz dans le matériau, les conditions aux limites les plus naturelles sur les températures sont pour le solide la température de la face exposée au rayonnement solaire et pour le gaz la température d'entrée (soit la température ambiante)

$$
\begin{aligned}
& T=T_{0}=\text { cte } \\
& \theta=\theta_{\mathrm{a}}=\text { cte }
\end{aligned}
$$

à la sortie du gaz, les conditions aux limites sont prises sur les gradients de température

$$
\operatorname{grad} T=0=\operatorname{grad} \theta
$$

- dans le cas contre-courant

il y a uniquement une inversion des conditions portant sur le gaz entre l'entrée et la sortie 
à l'entrée du gaz

$$
\begin{gathered}
\theta=\theta_{\mathrm{a}}=\text { cte } \\
\operatorname{grad} T=0
\end{gathered}
$$

à la sortie du gaz

$$
\begin{gathered}
T=T_{0}=\text { cte } \\
\operatorname{grad} \theta=0 .
\end{gathered}
$$

3. Développement des équations pour chacune des géométries. - 3.1 RAPPEL DU CAS « RECTANGULAIRE». - Nous rappelons ici les équations étudiées par G. Olalde, M. Daguenet et J. L. Peube [1] ainsi que leur solution analytique. Cas co-courant :

Le système différentiel constitué des équations (1)(2) complétées des conditions aux limites (5)-(6) s'écrit sous forme adimensionnelle en posant

$$
\begin{gathered}
T^{+}=\frac{T-\theta_{\mathrm{a}}}{T_{0}-\theta_{\mathrm{a}}} ; \quad \theta^{+}=\frac{\theta-\theta_{\mathrm{a}}}{T_{0}-\theta_{\mathrm{a}}} ; \quad x^{+}=\frac{x}{D} \\
\frac{\mathrm{d}^{4} T^{+}}{\mathrm{d} x^{+4}}-\frac{\operatorname{Pr} R e}{\xi} \frac{\mathrm{d}^{3} T^{+}}{\mathrm{d} x^{+3}}-6 N u\left(\frac{1-\xi}{\xi}+\frac{\lambda_{\mathrm{f}}}{\lambda_{\mathrm{s}}^{*}}\right) \frac{\mathrm{d}^{2} T^{+}}{\mathrm{d} x^{+2}}+ \\
+\frac{6 \lambda_{\mathrm{f}}}{\xi \lambda_{\mathrm{s}}^{*}} \operatorname{RePr} N u \frac{\mathrm{d} T^{+}}{\mathrm{d} x^{+}}=0 \\
\theta^{+}=T^{+}-\frac{\lambda_{\mathrm{s}}^{*}}{6 N u \lambda_{\mathrm{f}}} \frac{\mathrm{d}^{2} T^{+}}{\mathrm{d} x^{+2}}
\end{gathered}
$$

avec

$$
\begin{aligned}
& \text { en } x^{+}=0 \quad \theta^{+}=0 ; \quad T^{+}=1 \\
& \text { en } x^{+}=\frac{L}{D}=L^{+} \quad \frac{\mathrm{d} \theta^{+}}{\mathrm{d} x^{+}}=0=\frac{\mathrm{d} T^{+}}{\mathrm{d} x^{+}} .
\end{aligned}
$$

La solution de ces équations est une solution analytique de la forme :

$$
\begin{aligned}
T^{+}=A_{0}+A_{1} \mathrm{e}^{p_{1} x^{+}}+A_{2} \mathrm{e}^{p_{2} x^{+}}+A_{3} \mathrm{e}^{p_{3} x^{+}} \\
\theta^{+}=A_{0}+a_{1} A_{1} \mathrm{e}^{p_{1} x^{+}}+a_{2} A_{2} \mathrm{e}^{p_{2} x^{+}}+ \\
+a_{3} A_{3} \mathrm{e}^{p_{3} x^{+}} .
\end{aligned}
$$

Les coefficients $A_{0}, A_{1}, A_{2}$ et $A_{3}$ sont déterminés par les conditions aux limites.

La recherche des racines $p_{1}, p_{2}$ et $p_{3}$ s'effectue numériquement par la méthode de Bairstow.

\section{Cas contre-courant :}

Le cas contre-courant est résolu de manière analogue. Il est cependant plus commode de poser les rapports adimensionnels suivants :

$$
T^{+}=\frac{T-T_{0}}{\theta_{\mathrm{a}}-T_{0}} ; \quad \theta^{+}=\frac{\theta-T_{0}}{\theta_{\mathrm{a}}-T_{0}} ; \quad x^{+}=\frac{x}{D} .
$$

Les auteurs ont ainsi obtenu les profils de température adimensionnels $T^{+}=T^{+}\left(x^{+}\right)$pour le solide et $\theta^{+}=\theta^{+}\left(x^{+}\right)$pour le gaz en fonction de la distance axiale à la face frappée par le rayonnement incident.
3.2 CAS CYlindrique. - Cas co-courant : Le système différentiel des équations (1)-(2) complété des conditions aux limites (5)-(6) se développe en posant les rapports adimensionnels :

$$
\begin{gathered}
T^{+}=\frac{T-\theta_{\mathrm{a}}}{T_{0}-\theta_{\mathrm{a}}} ; \quad \theta^{+}=\frac{\theta-\theta_{\mathrm{a}}}{T_{0}-\theta_{\mathrm{a}}} ; \quad r^{+}=\frac{r}{D} \\
r^{+} \frac{\mathrm{d}^{2} T^{+}}{\mathrm{d} r^{+2}}+\frac{\mathrm{d} T^{+}}{\mathrm{d} r^{+}}-6 N u \frac{\lambda_{\mathrm{f}}}{\lambda_{\mathrm{s}}^{*}} r^{+}\left(T^{+}-\theta^{+}\right)=0 \\
r^{+} \frac{\mathrm{d}^{2} \theta^{+}}{\mathrm{d} r^{+2}}+\frac{\mathrm{d} \theta^{+}}{\mathrm{d} r^{+}}-\frac{P r R e}{\xi} r^{+} \frac{\mathrm{d} \theta^{+}}{\mathrm{d} r^{+}}+ \\
+6\left(\frac{1-\xi}{\xi}\right) N u r^{+}\left(T^{+}-\theta^{+}\right)=0
\end{gathered}
$$

avec

$$
\begin{array}{lll}
\text { en } \quad r^{+}=\frac{r_{0}}{D}=r_{0}^{+} & \theta^{+}=0 ; \quad T^{+}=1 \\
\text { en } \quad r^{+}=\frac{r_{\mathrm{s}}}{D}=r_{\mathrm{s}}^{+} & \frac{\mathrm{d} T^{+}}{\mathrm{d} r^{+}}=0=\frac{\mathrm{d} \theta^{+}}{\mathrm{d} r^{+}}
\end{array}
$$

- Cas contre-courant :

$$
\begin{aligned}
& T^{+}=\frac{T-T_{0}}{\theta_{\mathrm{a}}-T_{0}} ; \quad \theta^{+}=\frac{\theta-T_{0}}{\theta_{\mathrm{a}}-T_{0}} ; \quad r^{+}=\frac{r}{D} \\
& r^{+} \frac{\mathrm{d}^{2} T^{+}}{\mathrm{d} r^{+2}}+\frac{\mathrm{d} T^{+}}{\mathrm{d} r^{+}}-6 N u \frac{\lambda_{\mathrm{f}}}{\lambda_{\mathrm{s}}^{*}} r^{+}\left(T^{+}-\theta^{+}\right)=0 \\
& r^{+} \frac{\mathrm{d}^{2} \theta^{+}}{\mathrm{d} r^{+2}}+\frac{\mathrm{d} \theta^{+}}{\mathrm{d} r^{+}}+\frac{P r R e}{\xi} r^{+} \frac{\mathrm{d} \theta^{+}}{\mathrm{d} r^{+}}+ \\
& +6\left(\frac{1-\xi}{\xi}\right) N u r^{+}\left(T^{+}-\theta^{+}\right)=0
\end{aligned}
$$

avec

$$
\begin{array}{lll}
\text { en } \quad r^{+}=r_{0}^{+} & T^{+}=0 ; \frac{\mathrm{d} \theta^{+}}{\mathrm{d} r^{+}}=0 \\
\text { en } \quad r^{+}=r_{\mathrm{s}}^{+} & \theta^{+}=1 ; \frac{\mathrm{d} T^{+}}{\mathrm{d} r^{+}}=0 .
\end{array}
$$

3.3 CAS SPHÉRIQUE. - Quant au cas sphérique, en utilisant les mêmes rapports adimensionnels pour les températures et le rapport

$$
R^{+}=\frac{R}{D}
$$

pour la coordonnée d'espace, nous obtenons les systèmes différentiels :

- Cas co-courant :

$$
R^{+} \frac{\mathrm{d}^{2} T^{+}}{\mathrm{d} R^{+2}}+2 \frac{\mathrm{d} T^{+}}{\mathrm{d} R^{+}}-6 N u \frac{\lambda_{\mathrm{f}}}{\lambda_{\mathrm{s}}^{*}} R^{+}\left(T^{+}-\theta^{+}\right)=0
$$

$$
\begin{aligned}
R^{+} \frac{\mathrm{d}^{2} \theta^{+}}{\mathrm{d} R^{+2}} & +2 \frac{\mathrm{d} \theta^{+}}{\mathrm{d} R^{+}}-\frac{P r R e}{\xi} R^{+} \frac{\mathrm{d} \theta^{+}}{\mathrm{d} R^{+}}+ \\
& +6\left(\frac{1-\xi}{\xi}\right) N u R^{+}\left(T^{+}-\theta^{+}\right)=0
\end{aligned}
$$


avec

$$
\begin{aligned}
& \text { en } R^{+}=\frac{R_{0}}{D}=R_{0}^{+} \quad \theta^{+}=0 ; \quad T^{+}=1 \\
& \text { en } R^{+}=\frac{R_{\mathrm{s}}}{D}=R_{\mathrm{s}}^{+} \quad \frac{\mathrm{d} T^{+}}{\mathrm{d} R^{+}}=0=\frac{\mathrm{d} \theta^{+}}{\mathrm{d} R^{+}} \text {. }
\end{aligned}
$$

\section{- Cas contre-courant :}

$R^{+} \frac{\mathrm{d}^{2} T^{+}}{\mathrm{d} R^{+2}}+2 \frac{\mathrm{d} T^{+}}{\mathrm{d} R^{+}}-6 N u \frac{\lambda_{\mathrm{f}}}{\lambda_{\mathrm{s}}^{*}} R^{+}\left(T^{+}-\theta^{+}\right)=0$

$$
\begin{aligned}
R^{+} \frac{\mathrm{d}^{2} \theta^{+}}{\mathrm{d} R^{+2}} & +2 \frac{\mathrm{d} \theta^{+}}{\mathrm{d} R^{+}}+\frac{\operatorname{Pr} R e}{\xi} R^{+} \frac{\mathrm{d} \theta^{+}}{\mathrm{d} R^{+}}+ \\
& +6\left(\frac{1-\xi}{\xi}\right) N u R^{+}\left(T^{+}-\theta^{+}\right)=0
\end{aligned}
$$

avec

$$
\begin{array}{lll}
\text { en } \quad R^{+}=R_{0}^{+} & T^{+}=0 ; \frac{\mathrm{d} \theta^{+}}{\mathrm{d} R^{+}}=0 \\
\text { en } \quad R^{+}=R_{\mathrm{s}}^{+} & \theta^{+}=1 ; \frac{\mathrm{d} T^{+}}{\mathrm{d} R^{+}}=0 .
\end{array}
$$

Reíarque. - L'équation de continuité

$$
\operatorname{div}(\rho \mathbf{v})=0
$$

entraîne pour :

- le cas rectangulaire : une vitesse constante donc un nombre de Reynolds constant tout au long du passage du gaz dans le milieu poreux;

- le cas cylindrique : une vitesse inversement proportionnelle au rayon d'où un nombre de Reynolds que nous définissons de la façon suivante :

Cas co-courant :

$$
R_{\mathrm{e}}=\left(R_{\mathrm{e}}\right)_{0} \frac{r_{0}}{r}
$$

Cas contre-courant :

$$
R_{\mathrm{e}}=\left(R_{\mathrm{e}}\right)_{\mathrm{s}} \frac{r_{\mathrm{s}}}{r} .
$$

- Le cas sphérique : une vitesse inversement proportionnelle au carré du rayon d'où un nombre de Reynolds que nous définissons comme suit :

Cas co-courant :

$$
R_{\mathrm{e}}=\left(R_{\mathrm{e}}\right)_{0} \frac{R_{0}^{2}}{R^{2}} .
$$

Cas contre-courant

$$
R_{\mathrm{e}}=\left(R_{\mathrm{e}}\right)_{\mathrm{s}} \frac{R_{\mathrm{s}}^{2}}{R^{2}}
$$

4. Méthode numérique. - Pour résoudre les systèmes différentiels que nous nous sommes proposés d'intégrer, nous avons utilisé une méthode de RungeKutta d'ordre 5 associée à une méthode de double tir corrigé.

Cette méthode nous a permis de résoudre dans un premier temps les équations du cas rectangulaire et ainsi de vérifier les résultats de G. Olalde, M. Daguenet et $\mathrm{J}$. L. Peube [1] et dans un deuxième temps de résoudre les équations des cas cylindrique et sphérique.

L'ensemble des calculs a été effectué sur I.B.M. 370 au C.I.R.C.E. Nous n'avons rencontré aucune difficulté particulière dans la résolution des systèmes. La méthode de tir corrigé utilisée convergeait assez rapidement : après quatre tirs la précision souhaitée $\left(10^{-5}\right)$ était atteinte.

5. Résultats. - La méthode numérique utilisée permet de calculer les profils de températures dans le solide et dans le gaz en fonction de la distance radiale à la face intérieure de la cavité (face chaude).

Les figures 2 et 3 représentent précisément ces profils dans les cas de géométries cylindrique et

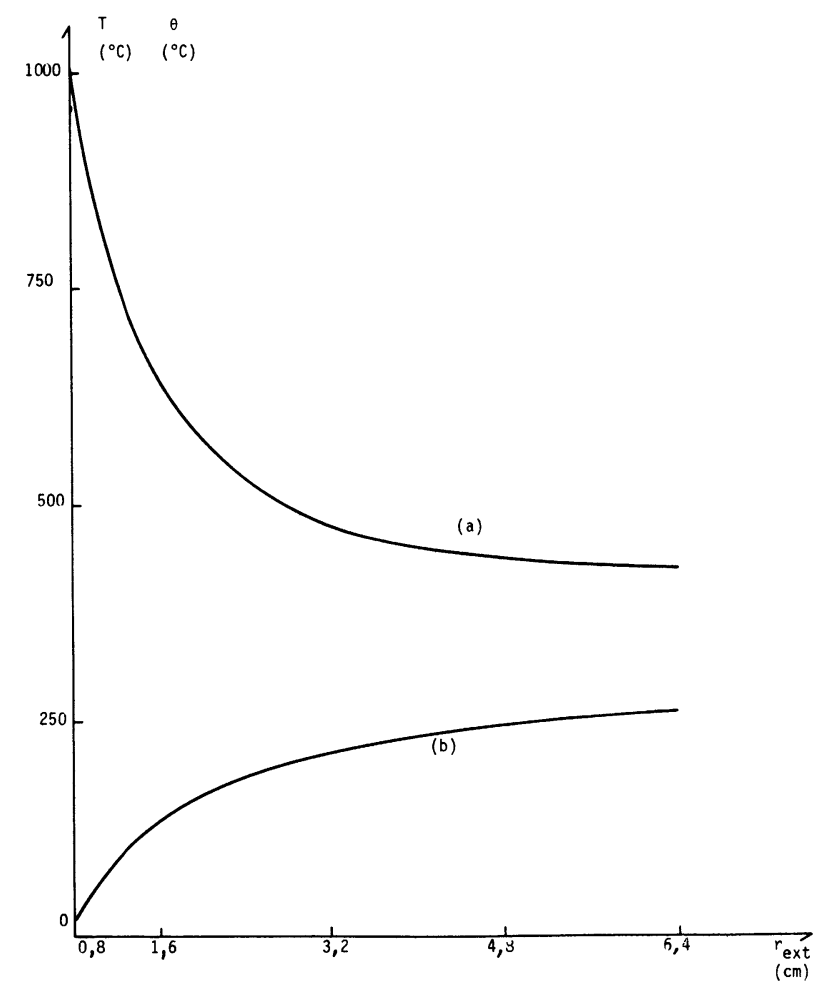

Fig. 2. - Cas cylindrique co-courant. Courbe a) Variation de la température dans le solide en fonction de la distance radiale à la face chaude. Courbe $b$ ) Variation de la température dans le gaz en fonction de la distance radiale à la face chaude.

[Co-current cylindrical case. Curve a) Variation of the temperature in the solid as a function of the radial distance to the hot solid front. Curve $b$ ) Variation of the temperature in the gas as a function of the radial distance to the hot solid front.] 


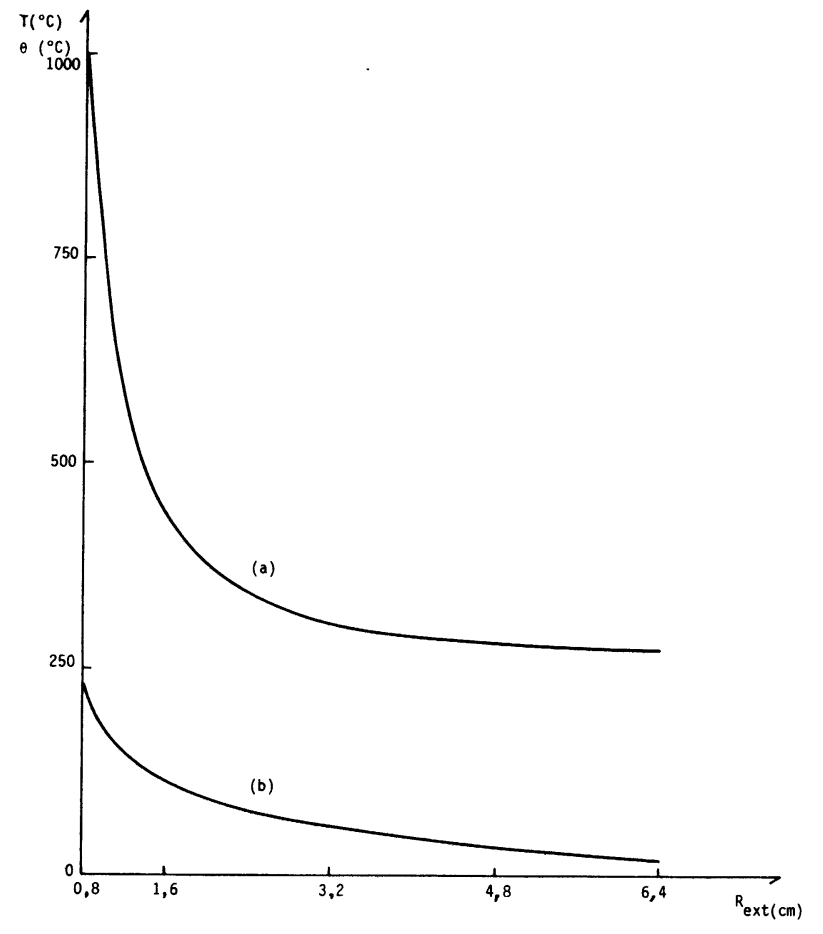

Fig. 3. - Cas sphérique contre-courant. Courbe a) Variation de la température dans le solide en fonction de la distance radiale à la face chaude. Courbe $b$ ) Variation de la température dans le gaz en fonction de la distance radiale à la face chaude.

[Counter-current spheric case. Curve $a$ ) Variation of the temperature in the solid as a function of the radial distance to the hot solid front. Curve $b$ ) Variation of the temperature in the gas as a function of the radial distance to the hot solid front.]

sphérique pour une face intérieure de la cavité de température $T_{0}=1000^{\circ} \mathrm{C}$.

Ces profils de températures dépendent d'une série de paramètres propres à l'écoulement et aux deux phases du système. Ce sont :

1) Les paramètres d'écoulement :

a) vitesse d'entrée du gaz dans le matériau,

b) sens de l'écoulement : co-courant ou contrecourant.

2) Les paramètres du gaz :

a) propriétés physiques du gaz (par l'intermédiaire du nombre de Prandtl).

Dans le cadre de cette publication, nous étudierons uniquement le cas d'un écoulement d'air (nombre de Prandtl $: P r \simeq 0,7$ à $20^{\circ} \mathrm{C}$ ).

3) Les paramètres du solide :

a) Propriétés géométriques :

- macroscopiques : - rayons intérieur et extérieur de l'échantillon,

- microscopiques :

- porosité,

- diamètre des particules. b) Propriétés physiques :

- conductivité thermique apparente.

5.1 INFLUENCE DE LA VITESSE D'ENTRÉE DU GAZ DANS L'ÉCHANTILLON. - La température de sortie du gaz varie en fonction de sa vitesse d'entrée dans l'échantillon, tous autres paramètres étant fixes par ailleurs.

Un exemple de telles variations est représenté graphiquement figure 4 dans le cas d'un écoulement co-courant en cavité cylindrique.

On constate que la température de sortie du gaz passe par un maximum lorsque la vitesse augmente.

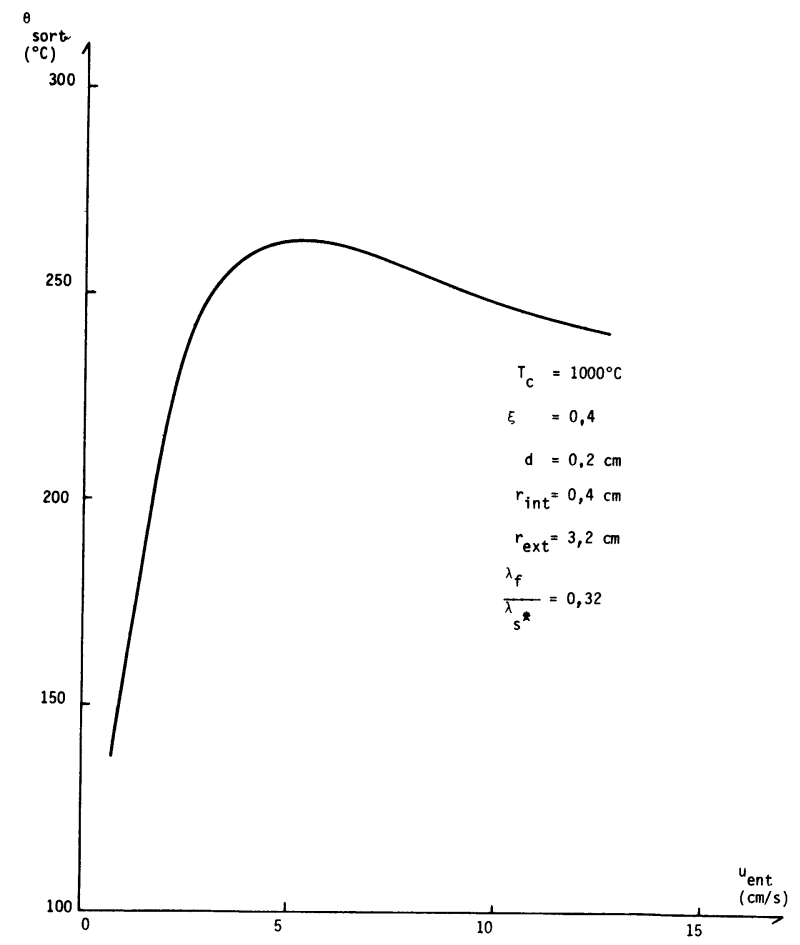

Fig. 4. - Variation de la température de sortie de l'air en fonction de sa vitesse d'entrée : cas cylindrique co-courant.

[Variation of the output temperature of the air as a function of the input speed : co-current cylindrical case.]

\subsection{RECHERCHE DES TEMPÉRATURES MAXIMALES. -} L'étude de la température de sortie du gaz en fonction de sa vitesse d'entrée montre que pour un rayon extérieur donné de l'échantillon tous autres paramètres étant fixes par ailleurs, il existe une vitesse optimale pour laquelle cette température est maximale.

Cela nous conduit à rechercher les variations des températures maximales de sortie du gaz en fonction $\mathrm{du}$ rayon extérieur et les valeurs optimales correspondantes de la vitesse d'entrée du gaz.

Les figures 5 et 6 représentent ces variations sous forme adimensionnelle dans des cas d'écoulement co-courant et contre-courant en cavité cylindrique. 


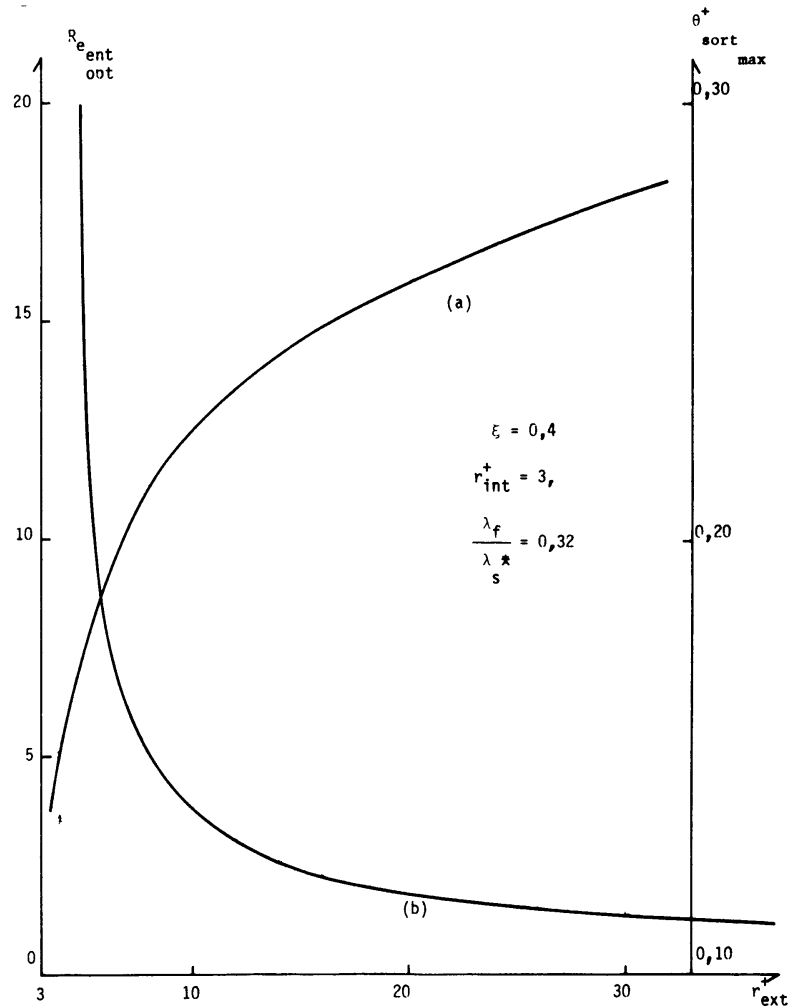

Fig. 5. - Cas cylindrique co-courant. Courbe a) Variation de la température de sortie maximale de l'air en fonction du rayon extérieur de l'échantillon. Courbe $b$ ) Variation du nombre de Reynolds optimal d'entrée de l'air en fonction du rayon extérieur de l'échantillon.

[Co-current cylindrical case. Curve $a$ ) Variation of the maximal output temperature of the air as a function of the exterior radius. Curve $b$ ) Variation of the optimal input Reynolds number of the air as a function of the exterior radius.]

5.3 SENS De L'ÉCOULEMENT : COMPARAISON « COCOURANT »-« CONTRE-COURANT ». - Si l'on compare les températures maximales de sortie du gaz obtenues en écoulements co-courant et contre-courant pour différents rayons extérieurs de l'échantillon, on constate que les températures obtenues en écoulement contre-courant sont supérieures à celles obtenues en écoulement co-courant. L'écart entre ces températures croît avec le rayon extérieur de l'échantillon. Toutefois cet écart reste faible et ne permet pas de privilégier un sens d'écoulement par rapport à l'autre.

On notera cependant que les vitesses d'entrée du gaz nécessaires pour obtenir ces températures sont bien plus faibles dans le cas contre-courant.

\subsection{INFLUENCE DU RAYON EXTÉRIEUR DE L'ÉCHAN-} TILLON. - La figure 7 donne sous forme adimensionnelle les résultats de la variation de température de sortie du gaz en fonction du rayon extérieur de l'échantillon dans le cas d'un écoulement co-courant en cavité cylindrique.

On constate que la température de sortie du gaz tend vers une limite maximale. Cette limite est atteinte

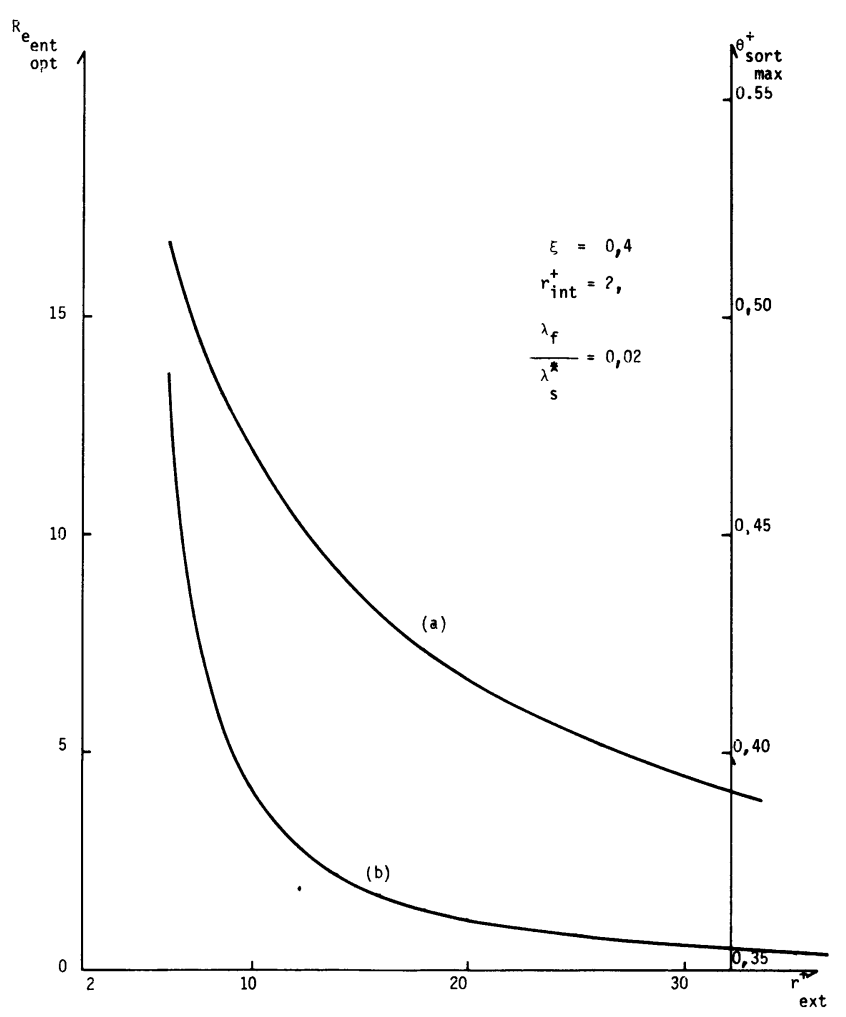

Fig. 6. - Cas cylindrique contre-courant. Courbe a) Variation de la température de sortie maximale de l'air en fonction du rayon extérieur de l'échantillon. Courbe b) Variation du nombre de Reynolds optimal d'entrée de l'air en fonction du rayon extérieur de l'échantillon.

[Counter-current cylindrical case. Curve a) Variation of the maximal output temperature of the air as a function of the exterior radius. Curve $b$ ) Variation of the optimal input Reynolds number of the air as a function of the exterior radius.]

plus ou moins lentement suivant la valeur de la vitesse d'entrée du gaz et suivant la conductivité thermique apparente du solide.

\section{5 INFLUENCE DE LA CONDUCTIVITÉ APPARENTE DU} SOLIDE. - La conductivité thermique apparente du solide est calculée suivant un modèle tenant compte du rayonnement entre grains, des contacts solides et du voisinage de ces contacts dans lequel le gaz peut être considéré comme immobile. Nous utilisons [6]

$$
\lambda_{\mathrm{s}}^{*}=\frac{(1-\xi) \beta^{\prime} \lambda_{\mathrm{s}}}{\varphi^{\prime} \frac{\lambda_{\mathrm{s}}}{\lambda_{\mathrm{f}}}+D \frac{h_{\mathrm{rs}}}{\lambda_{\mathrm{f}}}}
$$

$\beta^{\prime}$ et $\varphi^{\prime}$ sont des longueurs adimensionnelles caractéristiques ;

$\lambda_{\mathrm{s}}$ est la conductivité thermique des particules solides;

$h_{\mathrm{rs}}$ est le coefficient de transfert de chaleur par rayonnement thermique de surface solide à surface solide. 


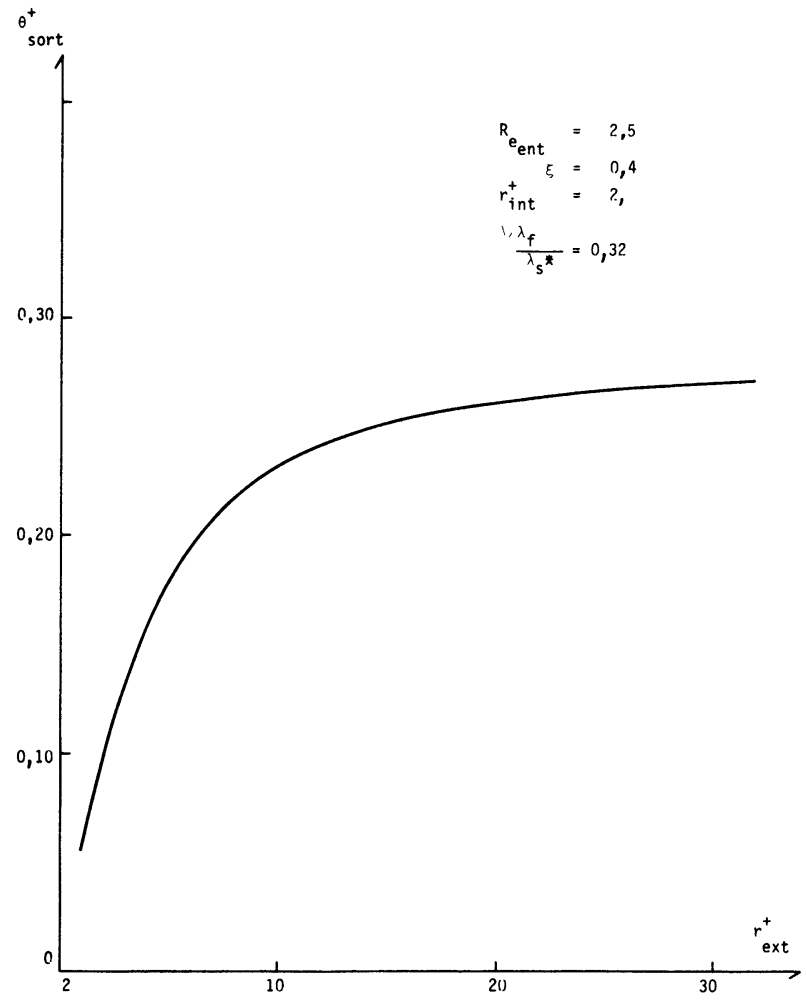

Fig. 7. - Variation de la température de sortie de l'air en fonction du rayon extérieur de l'échantillon : cas cylindrique co-courant.

[Variation of the output temperature of the air as a function of the exterior radius : co-current cylindrical case.]

L'étude de la température de sortie du gaz en fonction de la conductivité thermique apparente $\mathrm{du}$ solide montre que cette température augmente lorsque le rapport des conductivités $\lambda_{\mathrm{f}} / \lambda_{\mathrm{s}}^{*}$ (Fig. 8) diminue.

L'influence d'autres paramètres du système : porosité, diamètre des particules, rayon intérieur de la cavité, nature du gaz peut également être déterminée [7].

6. Bilan énergétique. - Nous avons calculé les profils de température dans le solide et dans le gaz pour divers paramètres du système en prenant comme donnée du problème la température de la face du solide exposée au rayonnement solaire. Nous voudrions connaître maintenant la quantité d'énergie rayonnante nécessaire pour obtenir cette température et les profils correspondants.

Pour cela, nous devons écrire le bilan d'énergie global sur l'échantillon.

Dans un premier temps nous écrivons ce bilan dans le cas de la géométrie rectangulaire étudiée par G. Olalde, M. Daguenet et J. L. Peube [1].

Nous supposons :

- le flux incident de rayonnement uniforme,

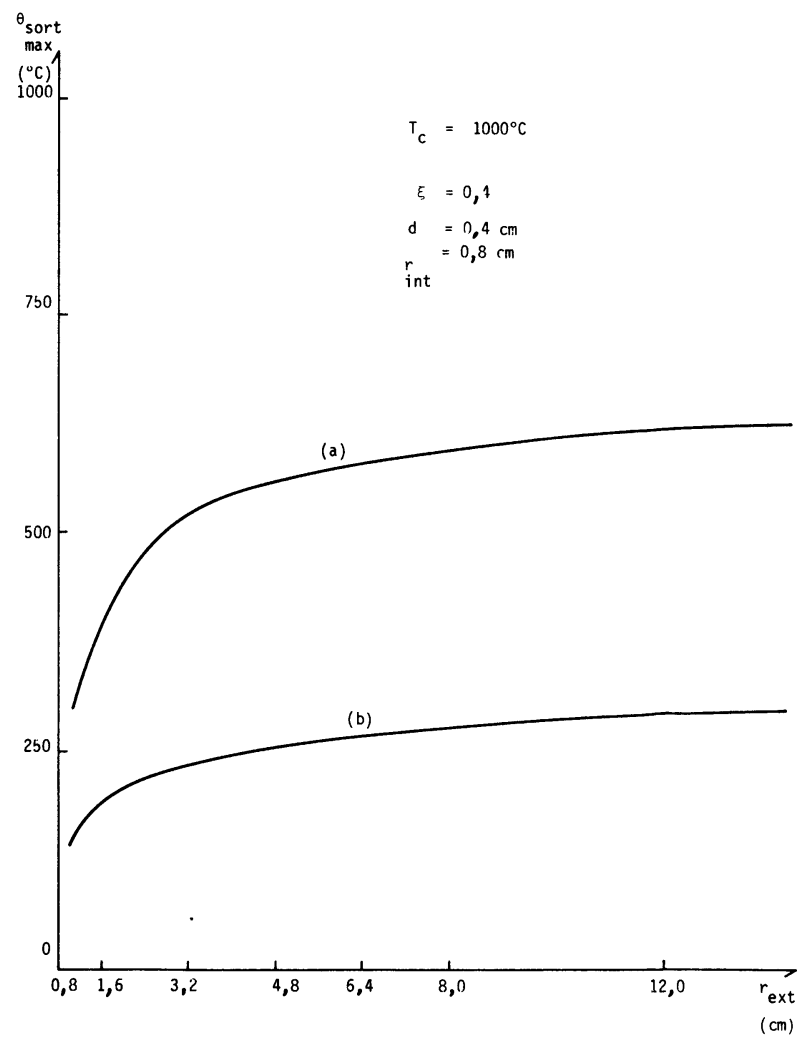

Fig. 8. - Cas cylindrique contre-courant. Variation de la température maximale de sortie de l'air en fonction du rayon extérieur de l'échantillon pour diverses conductivités apparentes du solide. Courbe a) $\lambda_{\mathrm{f}} / \lambda_{\mathrm{s}}^{*}=0,02 ;$ Courbe $\left.b\right) \lambda_{\mathrm{f}} / \lambda_{\mathrm{s}}^{*}=0,32$.

[Counter-current cylindrical case : variation of the maximal output temperature of the air as a function of the exterior radius for different apparent conductivities of the solid. Curve $a) \lambda_{\mathrm{f}} / \lambda_{\mathrm{s}}^{*}=0.02$; Curve $b$ ) $\lambda_{\mathrm{f}} / \lambda_{\mathrm{s}}^{*}=0.32$.]

- les rayons solaires perpendiculaires à la section avant de l'échantillon,

- les particules solides du milieu grises. Le bilan global s'écrit :

$\alpha \varphi=\varepsilon \sigma\left(T_{0}^{4}-\theta_{\mathrm{a}}^{4}\right)-\lambda_{\mathrm{s}}^{*}\left(\frac{\mathrm{d} T}{\mathrm{~d} x}\right)_{0}+\varepsilon \sigma\left(T_{\mathrm{L}}^{4}-\theta_{\mathrm{a}}^{4}\right)$

où $T_{\mathrm{L}}$ est la température de la section arrière de l'échantillon. Dans le cas des cavités (cylindrique ou sphérique) en supposant que le flux incident arrive de façon isotrope sur l'ouverture de la cavité nous écrivons :

$$
\begin{aligned}
\alpha_{\mathrm{a}} s \varphi=\varepsilon_{\mathrm{a}} s \sigma\left(T_{0}^{4}-\theta_{\mathrm{a}}^{4}\right)-\lambda_{\mathrm{s}}^{*} & S_{\mathrm{m}}\left(\frac{\mathrm{d} T}{\mathrm{~d} r}\right)_{r o}+ \\
& +\varepsilon \sigma s^{\prime}\left(T_{r_{\mathrm{s}}}^{4}-\theta_{\mathrm{a}}^{4}\right) .
\end{aligned}
$$

Dans le cas rectangulaire la surface exposée au rayonnement solaire ou surface éclairée $s$ et la surface par laquelle pénètre le gaz ou surface mouillée $s_{\mathrm{m}}$ sont confondues. Dans le cas de cavités cylindrique ou sphérique la surface éclairée est la surface d'ouverture de la cavité; la surface mouillée est la surface intérieure de la cavité. 


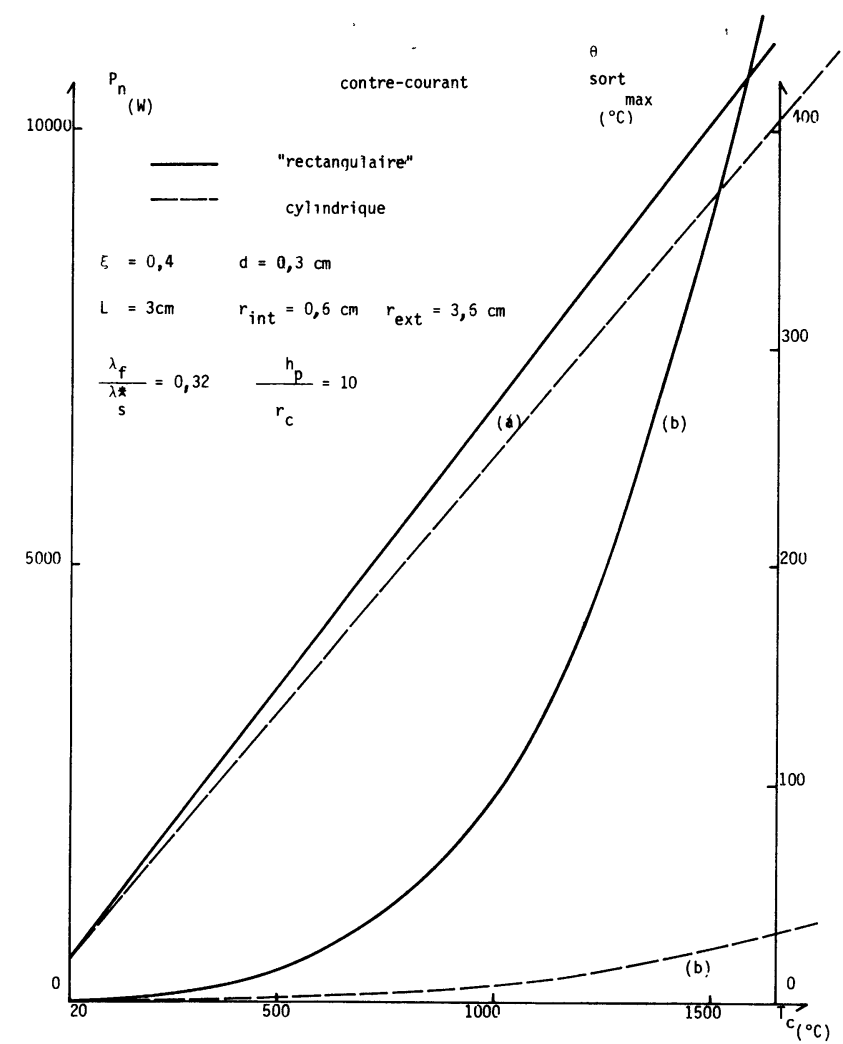

Fig. 9. - Comparaison géométrie rectangulaire-géométrie cylindrique contre-courant. Courbe $a$ ) Température maximale de sortie de l'air en fonction de la température chaude $T_{0}$ de la face exposée au rayonnement incident. Courbe $b$ ) Puissance nécessaire pour porter la face exposée au rayonnement à la température chaude $T_{0}$ en fonction de cette même température $T_{0}$.

[Comparison of the counter-current rectangular and cylindrical cases. Curve $a$ ) Maximal output temperature of the air as a function of the hot solid front temperature $T_{0}$. Curve $b$ ) Required power to obtain the hot solid front temperature $T_{0}$ as a function of this temperature $T_{0}$.]

Les facteurs d'absorption $\alpha_{\mathrm{a}}$ et d'émission $\varepsilon_{\mathrm{a}}$ sont des facteurs d'absorption et d'émission apparents donnés dans le cas de corps gris par l'expression [8] :

$$
\alpha_{\mathrm{a}}=\varepsilon_{\mathrm{a}}=\frac{\varepsilon_{+}\left[1+(1-\varepsilon)\left(s / S-\sin ^{2} \beta\right)\right]}{\varepsilon(1-s / S)+s / S}
$$

$S$ est la surface totale de la cavité ouverture comprise.

Les valeurs de $\sin ^{2} \beta$ et $s / S$ sont calculées en fonction du rapport $h / r_{\mathrm{c}}$ de la profondeur $h$ de la cavité au rayon $r_{\mathrm{c}}$ de son ouverture.

Il vient :

pour le cylindre :

$$
\sin ^{2} \beta=\frac{1}{1+\left(\frac{h}{r_{c}}\right)^{2}} ; \quad \frac{S}{S}=\frac{1}{2\left(1+\frac{h}{r_{c}}\right)}
$$

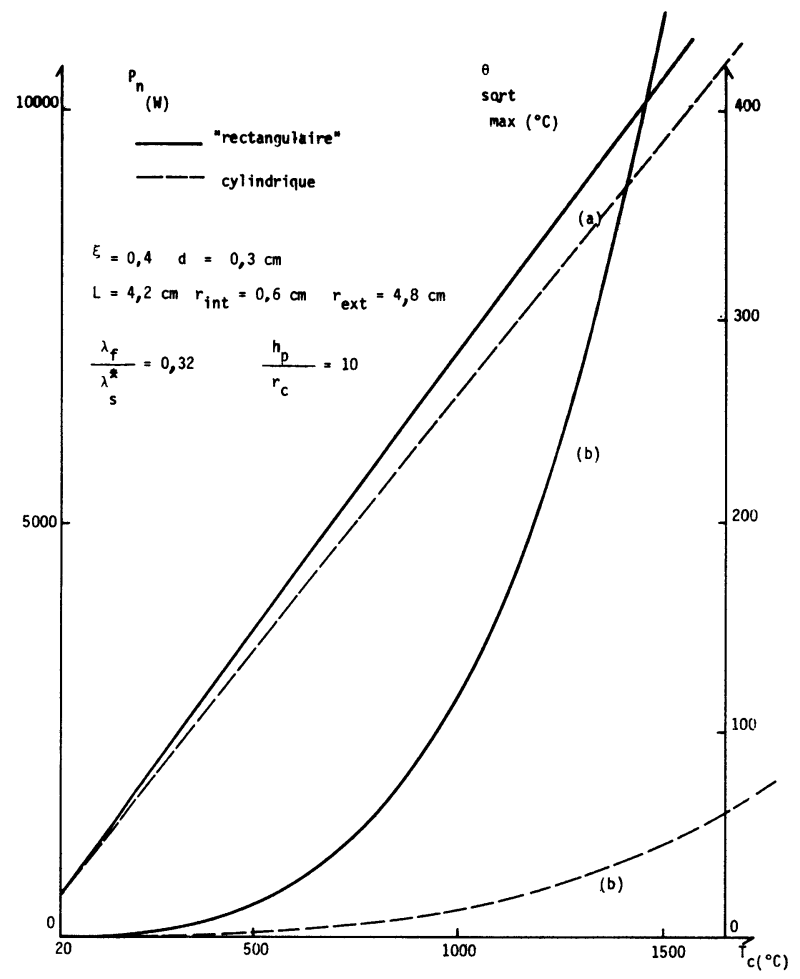

Fig. 10. - Comparaison géométrie rectangulaire-géométrie cylindrique co-courant. Courbe a) Température maximale de sortie de l'air en fonction de la température chaude $T_{0}$ de la face exposée au rayonnement incident. Courbe $b$ ) Puissance nécessaire pour porter la face exposée au rayonnement à la température chaude $T_{0}$ en fonction de cette même température $T_{0}$.

[Comparison of the co-current rectangular and cylindrical cases. Curve a) Maximal output temperature of the air as a function of the hot solid front temperature $T_{0}$. Curve $b$ ) Required power to obtain the hot solid front temperature $T_{0}$ as a function of this temperature $T_{0}$.]

pour la sphère

$$
\sin ^{2} \beta=\frac{1}{1+\left(\frac{h}{r_{\mathrm{c}}}\right)^{2}} ; \quad \frac{s}{S}=\frac{1}{2+\left(\frac{h}{r_{\mathrm{c}}}\right)^{2}} .
$$

Nous noterons la différence entre les surfaces d'émission des faces intérieures et extérieures de la cavité. La face intérieure émet par l'intermédiaire de la surface d'ouverture du corps alors que la face extérieure émet sur toute sa surface $s^{\prime}$.

Nous pouvons dès lors calculer, soit dans le cas rectangulaire, soit dans le cas des cavités cylindrique et sphérique, la puissance $P_{\mathrm{n}}=s \varphi$ nécessaire pour porter le matériau à une température $T_{0}$ donnée.

Sur les figures 9 et 10 , nous comparons les performances des cas rectangulaire et cylindrique, sur la figure 11 les performances des cas rectangulaire et sphérique et sur la figure 12 les performances des cas cylindrique et sphérique.

Si la température de sortie du gaz est, pour de 


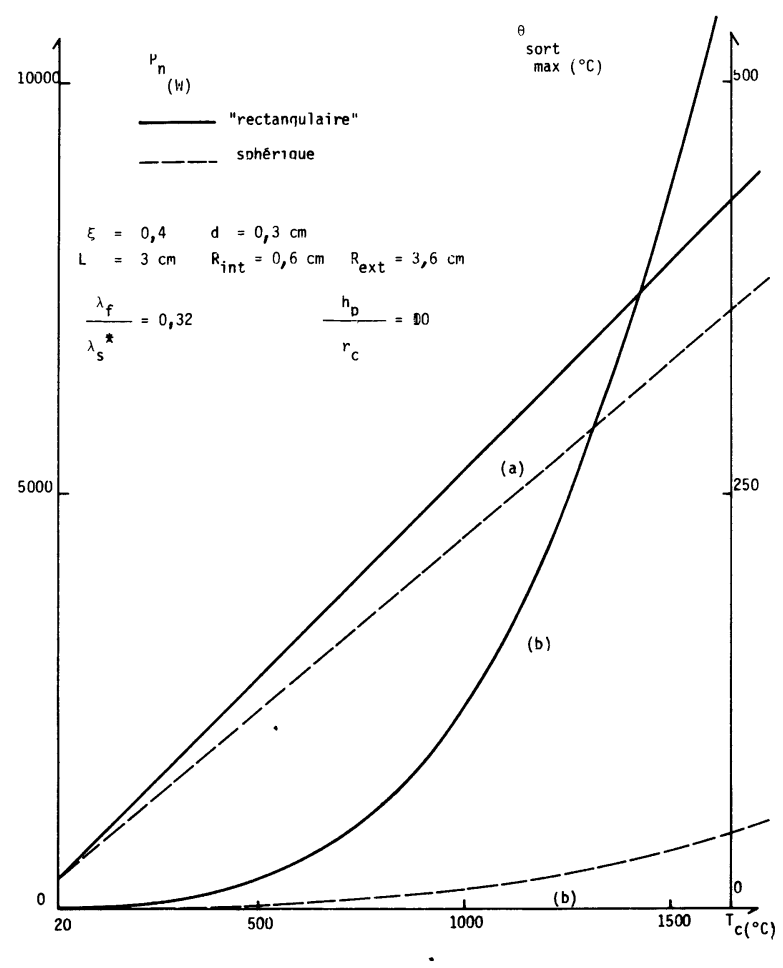

Fig. 11. - Comparaison géométrie rectangulaire-géométrie sphérique contre-courant. Courbe $a$ ) Température maximale de sortie de l'air en fonction de la température chaude $T_{0}$ de la face exposée au rayonnement incident. Courbe $b$ ) Puissance nécessaire pour porter la face exposée au rayonnement à la température chaude $T_{0}$ en fonction de cette même température $T_{0}$.

[Comparison of the counter-current rectangular and spheric cases. Curve a) Maximal output temperature of the air as a function of the hot solid front temperature $T_{0}$. Curve $b$ ) Required power to obtain the hot solid front temperature $T_{0}$ as a function of this temperature $T_{0}$.]

mêmes paramètres géométriques et physiques du matériau, meilleure dans le cas rectangulaire, elle nécessite pour son obtention une puissance très supérieure.

7. Conclusion. - Le programme de simulation numérique, grâce à sa souplesse et sa rapidité, permet d'étudier l'influence de tous les paramètres du système (propriétés physiques $\mathrm{du}$ gaz et du solide, propriétés géométriques $\mathrm{du}$ solide, caractéristiques

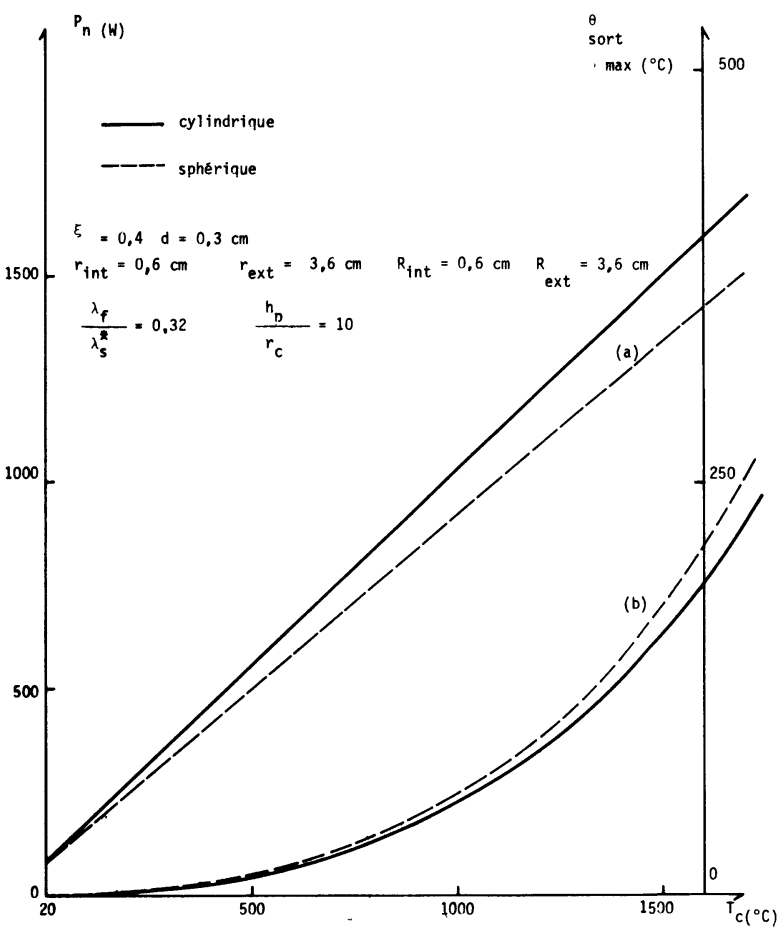

Fig. 12. - Comparaison géométrie cylindrique-géométrie sphérique contre-courant. Courbe $a$ ) Température maximale de sortie de l'air en fonction de la température chaude $T_{0}$ de la face exposée au rayonnement incident. Courbe $b$ ) Puissance nécessaire pour porter la face exposée au rayonnement à la température chaude $T_{0}$ en fonction de cette même température $T_{0}$.

[Comparison of the counter-current cylindrical and spheric cases. Curve $a$ ) Maximal output temperature of the air as a function of the hot solid front temperature $T_{0}$. Curve $b$ ) Required power to obtain the hot solid front temperature $T_{0}$ as a function of this temperature $T_{0}$.]

dynamiques de l'écoulement) et de traiter un grand nombre de géométries.

La comparaison des performances énergétiques du système étudié par G. Olalde, $M$. Daguenet et J. L. Peube avec celles des cavités plaide très nettement en faveur de ces dernières. Ce résultat était attendu puisque les pertes d'énergie par réémission sont beaucoup plus faibles pour une cavité que pour un plan. Par contre le choix de la cavité dépend plus des moyens à mettre en œuvre pour sa réalisation que de ses performances.

\section{Bibliographie}

[1] Olalde, G., Daguenet, M. et Peube, J. L., Etude théorique du chauffage d'un gaz dans un matériau poreux soumis à un rayonnement solaire concentré. Revue internationale des hautes températures et des réfractaires (sous presse).

[2] Beveridge, G. S. G. et Haughey, D. D., Axial heat transfer in packed beds. Stagnant beds between 20 and $750^{\circ} \mathrm{C}$. Int. J. Heat Mass Transfer 14 (1971) 1093.

[3] Argo, W. B. et Smith, J. M., Heat transfer in packed beds. Chem. Engineer. Prog. 49 (8) 443-451.

[4] Dumez, P., La conductivité thermique des matériaux pulvérulents et granulaires et ses méthodes de mesure en régime établi. Revue générale de thermique, $\mathrm{n}^{\circ} 55$, juillet 1966.
[5] Calvet, P., Echanges thermiques dans les lits poreux. I.F.C.E. Journées de la transmission de la chaleur (juin 1961).

[6] KuNI, D. et SMITH, J. M., Heat transfer characteristics of porous rocks. A.I.Ch.E. Journal 6 (1960) 71-78.

[7] Genneviève, F., Etude théorique du chauffage des gaz en milieu poreux par énergie solaire. Thèse de spécialité (17 novembre 1979, Université de Perpignan).

[8] Gouffe, A., Transmission de la chaleur par rayonnement : formules et données numériques, $2^{\mathrm{e}}$ édition revue et corrigée. Paris, Eyrolles, 1978 